\title{
Re-Examination of the Effect of the Stretch/Orientation-Induced Reduction of Friction under Equi-Biaxial Elongational Flow via Primitive Chain Network Simulation Using Two Definitions of Orientation Anisotropy
}

\author{
Keiko Takeda, Sathish Kumar Sukumaran, Masataka Sugimoto, Kiyohito Koyama and Yuichi MasubuchI ${ }^{*}{ }^{\dagger}$ \\ Graduate School of Organic Materials Science, Yamagata University, Yonezawa 992-8510, Japan \\ *Department of Materials Physics, Nagoya University, Furocho, Chikusa, Nagoya 464-8603, Japan
}

(Received : March 16, 2018)

\begin{abstract}
In this study, we examined the effect of the stretch/orientation-induced reduction of friction (SORF) between under uniaxial and equi-biaxial elongational flows using the primitive chain network (PCN) simulation with two definitions of orientation anisotropy under equi-biaxial elongational flow. We used two types of the orientation parameter $S\left(S_{1}\right.$ and $\left.S_{1 / 2}\right)$ under equi-biaxial elongational flow, giving rise to different values 1 and $1 / 2$ of $S$, as the limiting values for $S_{1}$ and $S_{1 / 2}$ along the elongational direction, respectively. We calculated the steady-state elongational viscosity of monodisperse polystyrene melts to observe the SORF, which should be effected at large strain rate by PCN simulation. Furthermore, we attempted molecular level analysis utilizing decoupling approximation. The difference between $S_{1}$ and $S_{1 / 2}$ was the critical strain rate of the SORF effect when using $S_{1 / 2}$. The friction decreased at the critical strain rate, which was comparable with the inverse of the Rouse relaxation time under uniaxial and equi-biaxial elongational viscosities. On the other hand, the friction under equi-biaxial elongational flow using $S_{1}$ decreased at earlier than that.
\end{abstract}

Key Words: Biaxial elongational flow / Orientation / Reduction of friction / Simulation

\section{INTRODUCTION}

Many molecular theories of entangled polymer liquids based on the tube model by Doi and Edwards ${ }^{1)}$ have been proposed for understanding of entangled molecular behavior. Many modifications of the tube model has been made for more precise prediction of polymer liquid behavior ${ }^{2-4)}$.

Nevertheless, molecular behavior under fast elongational flow has not been fully explained. It has been known that linear monodisperse polystyrene (PS) concentrated solutions exhibit the increase of steady-state elongational viscosity as increasing the strain rate $(\dot{\varepsilon})$ when the strain rate is higher than the inverse Rouse time ${ }^{5-7)}$, though PS melts show a decrease of the steady-state elongational viscosity ${ }^{8,9}$. Yaoita et $a l .{ }^{10)}$ focused on a flow-induced change of the monomeric friction coefficient $\zeta$ which was proposed by Ianniruberto $e t$ $a l .{ }^{11)}$ to solve the issue under the fast uniaxial elongation. They made the hypothesis that the monomeric friction coefficient $\zeta$ was changed by the extent of stretch and orientation of polymeric chains under high strain rates (stretch/orientation-induced reduction of monomeric friction: SORF). They

\footnotetext{
$\dagger$ Corresponding author.

E-mail : mas@mp.pse.nagoya-u.ac.jp

Tel: +81-52-789-2551;
}

evaluated how the SORF in the primitive chain network (PCN) simulation including finite extensible nonlinear elasticity $(\mathrm{FENE})^{12,13)}$ affects the elongational flow behavior. They mentioned that the entangled subchains become rapidly close to fully orientation and stretch limit, hence $\zeta$ significantly decreases and then the steady-state elongational viscosity exhibits the monotonic thinning under fast elongation for melts. Conversely, for solutions, the solvent governs an average orientational anisotropy of the polymer chains and remains it small, and a change of the friction $\zeta$ cannot be seen in the available range of the simulation, differing from the polymer melts. This in solutions leads to an increase of the viscosities as the strain rate increased. They determined the magnitude of SORF from the deformation stress decay after cessation of the fast uniaxial elongational flow for PS melts and solutions ${ }^{14)}$. The equation proposed by Yaoita $e$ al $^{10)}$ to express the SORF effect is as,

$$
\begin{aligned}
& \frac{\zeta\left(F^{\prime} \mathrm{SO}\right)}{\zeta(0)}=f_{\mathrm{FENE}} \frac{1}{(1+\beta) \gamma}\left[\beta+\frac{1}{2}\left\{1-\tanh \alpha\left(F^{\prime}{ }_{\mathrm{SO}}-F^{\prime}{ }^{*} \mathrm{SO}\right)\right\}\right]^{\gamma}, \\
& F_{\text {So }}^{\prime}=F_{\mathrm{SO}} f_{\mathrm{FENE}}, \\
& F_{\mathrm{SO}}=\tilde{\lambda}^{2} \bar{S} .
\end{aligned}
$$


where $F_{\text {SO }}$ is the so-called stretch-orientation order parameter, $\zeta$ is the segmental friction, and $\zeta(0)$ is the equilibrium value. $f_{\text {FENE }}$ is the finitely extensible nonlinear elastic factor defined as

$$
f_{\mathrm{FENE}}=\frac{1}{1-\widetilde{\lambda}^{2}} \text { with } \tilde{\lambda}=\lambda / \lambda_{\max }
$$

where $\tilde{\lambda}$ is the average stretch ratio normalized by the maximum stretch of entangled subchains $\left(\lambda_{\max }\right) \cdot \tilde{\lambda}^{2}$ is the ensemble average for all the segments of the entangled polymer chain. In Eq. 1, friction coefficient parameters, $\alpha(=20), \beta$ (= $\left.5 \times 10^{-9}\right), \gamma(=0.15)$ and the threshold value for the onset of friction reduction $F^{*}{ }_{\text {SO }}(=0.14)$ are determined via fitting of the experimental data ${ }^{10)} . \bar{S}$ is the average orientational anisotropy of the components defined as

$$
\bar{S}=\phi_{\mathrm{p}} S,
$$

where $\varphi_{\mathrm{p}}$ is the polymer volume fraction and is unity for polymer melts. $S$ is the polymer anisotropy of the subchain orientation vector $\mathbf{u}$ obtained from the eigenvalues of the orientation tensor $<\mathbf{u u}>$ where $\mathbf{u}$ denotes the unit bond vector and $<\ldots>$ denotes the ensemble average.

It is noteworthy that, the stretch-orientation order parameter $F_{\text {So }}$ proposed by Yaoita et al. ${ }^{10)}$ was determined from the uniaxial stress at the cessation of elongational flow. The deformation stress, with the decoupling approximation ${ }^{15)}$, can be written as

$$
\sigma=3 G_{0} f_{\mathrm{FENE}} \tilde{\lambda}^{2} S
$$

where $G_{0}$ is the unit modulus, $f_{\mathrm{FENE}}$ is the FENE factor, $\tilde{\lambda}^{2}$ the polymer stretch averaged for all the segments of an entangled polymer chain, and $S$ the orientation anisotropy. Note that Eq. 6 is compatible with the stress-optical rule $(\mathrm{SOR})^{16)}$ given that $f_{\mathrm{FENE}} \sim \tilde{\lambda}^{2} \sim 1$ under marginal flows. The possible reduction of entanglement due to convective constraint release is allowable because the orientation anisotropy of the segment that determined by SOR is proportional to the orientation anisotropy of the segment determined by the stress. According to Eq. 6, the first normal stress difference, which corresponds to the uniaxial stress, can be written as

$$
\sigma_{x x}-\sigma_{y y}=3 G_{0} f_{F E N E} \tilde{\lambda}^{2} S_{x x-y y}
$$

Here, $S_{x x-y y}$ is the orientational anisotropy defined under uniaxial deformation, and according to the stress-optical rule it should be written as

$$
S_{x x-y y}=\left\langle u_{x}^{2}\right\rangle-\left\langle u_{y}^{2}\right\rangle
$$

Yaoita et al. ${ }^{10)}$ utilized this orientational measure as their orientational order parameter, and obtained $F_{\text {SO }}^{\prime}=f_{\mathrm{FENE}} \tilde{\lambda}^{2} S_{x x-y y}$ from the experimentally observed $\sigma_{x x}-\sigma_{y y}$ at the flow cessation.

Following Yaoita et al. ${ }^{10)}$, one may extend SORF to the other type of deformations by considering an appropriate orientational order parameter. For equi-biaxial elongational flows, a possible formula is written as follows.

$$
S_{1 / 2}=\left(<u_{y}^{2}>+<u_{z}^{2}>\right) / 2-<u_{x}^{2}>
$$

This formula is rationalized according to the usual form of equi-biaxial stress. However, in this form, the maximum value of $S_{1 / 2}$ is $1 / 2$, which might inappropriately restrict the activation of SORF. According to such an idea, in our previous work $^{17)}$, we used the other form to set the maximum value of unity as

$$
S_{1}=\left(<u_{y}^{2}>+<u_{z}^{2}>\right)-2<u_{x}^{2}>
$$

with $S_{1}$, we simulated steady-state viscosities under equi-biaxial and uniaxial flows at various strain rates to study the effect of SORF ${ }^{17)}$. When we used the definition of the polymer anisotropy $S_{1}$ (Eq. 10) under equi-biaxial deformation, the onset of the SORF effect occurred at smaller strain rate than that of uniaxial deformation. Yaoita et al. ${ }^{10)}$ assumed that the friction coefficient of the Kuhn segment remains isotropic even under fast flow and can be characterized by the scalar factor, $\zeta$. That scalar quantity should be expressed regarding the scalar invariants of the tensor $<\mathbf{u u}>$. There are two orientation parameters, $S_{y y-x x}=<u_{y}^{2}>-<u_{x}^{2}>$ and $\left.S_{z z-x x}=<u_{z}^{2}>-<u_{x}^{2}\right\rangle$ for equi-biaxial flow, which affects to the stress. $S_{y y-x x}$ and $S_{z z-x x}$ are the same value. It is assumed that friction reduction may depend on not the average of orientation tensors but two orientation parameters for equi-biaxial elongational flow, it also depend on the $S_{1 / 2}$ value. We reviewed from these points the effect of two orientational anisotropy of polymer parameters (Eq. 9 and Eq. 10) on the SORF effect under equi-biaxial elongational flow and the resulting elongational flow behavior in this study, also compared with that of uniaxial deformation.

\section{MODEL}

We denote the primary aspect of the primitive chain 
network $(\mathrm{PCN})$ model $^{12)}$ because detailed description can be referred elsewhere ${ }^{10,17-19)}$. The polymer chains are connected through quasi-crosslinked points referred to as slip-links in 3-dimensional spaces. Consecutive strands (subchains) including two nodes which connect chains forming a group with other chains. Two subchains are linked to a slip-link so that four subchains diverge from each node. The motion of nodes (slip-links) is described by a Langevin-type equation of motion considering the force balance, subchain tension, osmotic force suppressing the density fluctuation, and random Brownian force. FENE is adopted as an elastic force with FENE-P approximation ${ }^{13)}$. The number of slip-links $Z$ fluctuates due to hooking and un-hooking processes between chains. The known molecular mechanisms for entangled polymers are considered: reptation ${ }^{20,21)}$, contour length fluctuation $^{22)}$, thermal ${ }^{23-25)}$ and convective ${ }^{2)}$ constraint release. In addition to these mechanisms, SORF is implemented according to Eqs. 1-5, where the orientational anisotropy is either Eq. 9 or 10. The parameters in Eq. 1 are assumed to be the same with those obtained from the stress relaxation under uniaxial deformations due to the absence of experimental data for equi-biaxial deformations.

\section{RESULTS}

Figure 1 shows the steady-state elongational viscosity $\eta_{E}$ divided by linear viscosity $\eta_{E(0)}$, the average orientation $\bar{S}$, square of normalized stretch by the maximum stretch $\tilde{\lambda}^{2}$, FENE factor $f_{F E N E}$, reduced segment number $Z / Z_{0}$ and reduced friction to the equilibrium value $\zeta\left(F_{S O}\right) / \zeta(0)$ as a function of Weissenberg number $W i$ defined with the Rouse relaxation time at equilibrium $\tau_{\mathrm{R}}^{0}, W i_{\mathrm{R}} \equiv \dot{\varepsilon} \tau_{\mathrm{R}}^{0}$, for equi-biaxial and uniaxial elongational flow of monodisperse melt in the steady-state, from top to bottom. The results with the definition of equi-biaxial orientation anisotropy $S_{1 / 2}$ (Eq. 9) as bold red curves, and thin blue curves are $S_{1}$ (Eq. 10) which we reported before ${ }^{17)}$. Thin black curves are for uniaxial flow. Solid curves are with SORF and dotted are without SORF.

Although we used same $W i_{\mathrm{R}}$ to compare uniaxial and equi-biaxial elongational viscosities, to be exact, when we compare them, we should use strain invariants because the strain rate is different. We will discuss this matter in other work.

At $W i_{\mathrm{R}}<0.1$, the steady-state elongational viscosity ratio $\eta / \eta_{0}\left(1^{\text {st }}\right.$ panel $)$ for uniaxial flow is almost flat, and $\eta / \eta_{0}$ for equi-biaxial flow decreases as increasing $W i_{\mathrm{R}}$, independent of the use of $S_{1 / 2}$ and $S_{1}$. In this region, it is clear that $\eta / \eta_{0}\left(1^{\text {st }}\right.$ panel) is dominated by the orientation ( $2^{\text {nd }}$ panel) because the squared reduced stretch and $f_{\mathrm{FENE}}$ remain unchanged in this area $\left(3^{\text {rd }}\right.$ and $4^{\text {th }}$ panels) for both flows. Although the orientational anisotropy of both flows are already induced, even at this low $W i_{\mathrm{R}}$ range, the growth rates of orientation anisotropy $S\left(S_{1}\right.$ and $\left.S_{1 / 2}\right)$ are weaker than that of uniaxial flow. In addition to this, the decreases of $Z / Z_{0}$ under equi-biaxial flows $\left(S_{1}\right.$ and $S_{1 / 2}$ ) are somewhat steeper. These induce the decrease of $\eta / \eta_{0}$ of equi-biaxial flows $\left(S_{1}\right.$ and $\left.S_{1 / 2}\right)$. Thus, under the uniaxial elongational flow, steeply increasing of $S\left(2^{\text {nd }}\right.$ panel $)$ and gradual decrease of $Z / Z_{0}\left(5^{\text {th }}\right.$ panel $)$ comparing with equi-biaxial flow corresponds to the constant value of $\eta / \eta_{0}\left(1^{\text {st }}\right.$ panel $)$ in this area.

At $0.1<W i_{\mathrm{R}}<1$, decrease rates of all $\eta / \eta_{0}$ curves become mild, and the curves almost level off. This mainly

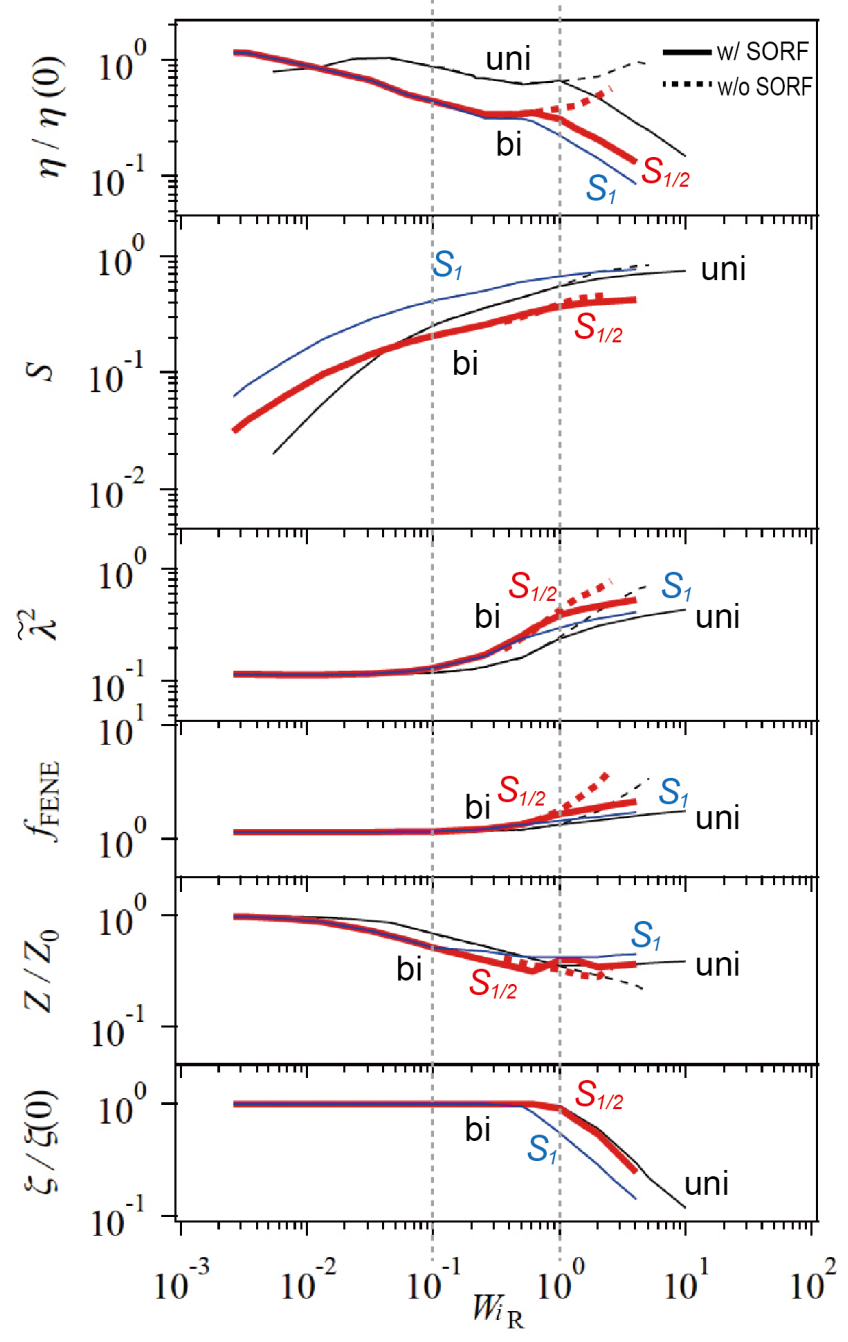

Fig. 1 Steady-state values for the reduced viscosity, orientation, squared reduced stretch, FENE factor, reduced segment number and reduced friction from top to bottom. The viscosity, segment number and friction are reduced with respect to the equilibrium values. The stretch $\tilde{\lambda}^{2}$ is normalized by the maximum stretch. Blue thin curves and black thin curves are for equi-biaxial and uniaxial deformations, respectively. Red bold curves are for equi-biaxial deformations with definition of Eq. 9. Blue thin curves are for equi-biaxial deformations with definition of Eq. 10. Solid and dotted curves are for the results with and without SORF. 
results from competing behavior of the chain stretch and orientation anisotropy. The stretch growth ( $3^{\text {rd }}$ panel) contributes to suppressing the decrease of $\eta / \eta_{0}$ under uniaxial and equi-biaxial flows despite the moderate growth of orientation anisotropy $S\left(2^{\text {nd }}\right.$ panel). In both cases of uniaxial and equi-biaxial $\left(S_{1 / 2}\right)$, the stretch/orientation order parameter $F_{\text {SO }}$ does not reach the threshold value $F^{\prime^{*}}$ so $(0.14)$ (Eq. 1), and friction reduction of uniaxial and equi-biaxial $\left(S_{1 / 2}\right)$ does not occur in this range $\left(6^{\text {th }}\right.$ panel). Contrary to this, for the equi-biaxial $\left(S_{1}\right)$ case, $\zeta / \zeta(0)\left(6^{\text {th }}\right.$ panel $)$ sharply decreases at around 0.5 of $W i_{\mathrm{R}}$, if SORF is considered. The developed and saturated orientation anisotropy of $S_{1}$ (Eq. 10) and the increase of stretch growth exceed the threshold value $F^{\prime *}$ so $(0.14)$ (Eq. 1), then a friction decrease is induced at the point, while $\zeta / \zeta(0)$ of uniaxial and equi-biaxial flow $\left(S_{1 / 2}\right)$ using Eq. 9 maintain unity.

At $W i_{\mathrm{R}} \geq 1$, if we consider SORF, $F^{\prime}$ so reach the threshold value $F^{*}$ so $(0.14)$ (Eq. 1 ), and friction reduction of uniaxial and equi-biaxial $\left(S_{1 / 2}\right)$ occur in this range $\left(6^{\text {th }}\right.$ panel). This friction reduction suppresses the growths of the stretch $\left(3^{\text {rd }}\right.$ panel) and FENE factor ( $4^{\text {th }}$ panel) for uniaxial and equi-biaxial $\left(S_{1 / 2}\right)$ elongational flow, both steady-state viscosities decrease as seen for equi-biaxial flow $\left(S_{1 / 2}\right)$. If we do not consider SORF, the growth of stretch and FENE increase with increasing $W i_{\mathrm{R}}$ for both flows and the steady-state viscosities increase. The simulation results which was obtained by taking account of $\zeta / \zeta(0)$ decrease at the high strain rates are in good agreement with the thinning behavior as seen in the experiments.

\section{DISCUSSION}

Desai and Larson $^{26)}$ predicted elongation thinning for entangled melts using the Doi-Edwards-Marrucci-Grizzutti (DEMG) model ${ }^{27)}$ modified by the friction reduction which Yaoita et al. proposed $^{10)}$, while modified DEMG model without the friction reduction failed to predict elongation thinning. Ianniruberto et al. ${ }^{28)}$ reported elongation thinning of the melts is due to the friction reduction using more simple equation than the original one from Yaoita et $a l^{10)}$. They performed nonequilibrium molecular dynamics simulation of styrene oligomers to obtain those diffusion coefficients and friction coefficients and compared with the data of the friction reduction $\zeta / \zeta(0)$ vs. the order parameter $S$ determined from the stress-optical data on PS melts ${ }^{29)}$. They also compared $\zeta / \zeta(0)$ vs. $S$ to the data of Yaoita et al. ${ }^{10)}$. They explained the reason of underprediction of the friction reduction $\zeta / \zeta(0)$ vs. the order parameter $S$ of Yaoita et $a l^{10)}$. was because of higher Rouse modes included. Addition to this, their data was calculated from steady-state stress-optical data, then it was not affected by time resolution ${ }^{28)}$. Their results suggest that the further consideration will be needed on the stress equation derived the reduction friction. Although these results confirm that the mechanism of friction reduction is essential to explain elongational behavior under fast flows, SORF is still controversial. Thus, it is crucial that to examine the effect of SORF under not only uniaxial but equi-biaxial and other elongational flow.

When we used $S_{1}$, friction reduction occurred below unity of $W i_{\mathrm{R}}$ due to the high value of orientation anisotropy $S_{1}$ comparing with uniaxial and equi-biaxial $\left(S_{1 / 2}\right)$, the stretch/ orientation order parameter $F_{\text {SO }}$ exceeded the threshold value of 0.14 (Eq. 1) at around 0.5 of $W i_{\mathrm{R}}$ if SORF on. In cases of equi-biaxial $\left(S_{1 / 2}\right)$ and uniaxial elongational flow, when SORF was considered, the stretch growth and FENE were suppressed, and friction decreased, consequently of this, the viscosities decreased at $W i_{\mathrm{R}} \geq 1$. Friction reduction occurred at same $W i_{\mathrm{R}}$ under uniaxial and equi-biaxial $\left(S_{1 / 2}\right)$ elongational flow, due to fully orientation limit for the uniaxial flow and equi-biaxial $\left(S_{1 / 2}\right)$ flow, respectively.

However, we have not compared these results with experimental equi-biaxial data because equi-biaxial elongational experiments for monodisperse polymer at large strain rate are not available. Furthermore, the SORF equation is empirical form derived from the experimental data of the stress relaxation after cessation of uniaxial elongational viscosities. We need more careful consideration whether we apply SORF to the different elongational flow to optimize the required parameters and investing polymer dynamics under various elongational flow manners. Indeed, as mentioned in the model description, we employed the strong assumption, in which the functional form of SORF and the parameters therein are common to those under uniaxial deformation. Experimental investigations, as well as simulation studies on this matter, are apparently required.

\section{CONCLUSION}

In this study, we compared the effect of the definition of orientation parameter $S$ on the stretch/orientation-induced reduction of friction (SORF) under uniaxial and equi-biaxial elongational flow via primitive chain network simulation using newly defined $S_{1 / 2}$ (Eq. 9) and previously reported $S_{1}$ (Eq. $10)$. We calculated the steady-state viscosity of the monodisperse polymer melts under equi-biaxial elongational flow in the wide strain rate range, and then examined molecular behavior by utilizing decoupling equation. We found that the 
effect of SORF under uniaxial and equi-biaxial elongational flow using equi-biaxial orientation definition $S_{1 / 2}$ was almost identical, and friction decreased at $W i_{\mathrm{R}} \geq 1$. Meanwhile, equi-biaxial elongational flow using the orientation definition $S_{1}$, SORF occurred at $W i_{\mathrm{R}}<1$. The differences in orientation anisotropy definitions affected $F_{\text {SO }}$ and changed critical $W i_{\mathrm{R}}$ for the onset of friction reduction under equi-biaxial elongation. To be fair, we emphasize that the results reported here be based on the strong assumption of the universality of SORF behavior for different deformation fields. For the validity of this assumption experimental and/or simulation studies are necessary.

\section{REFERENCES}

1) Doi M, Edwards SF, The Theory of Polymer Dynamics, Clarendon Press. Oxford, (1986).

2) Marrucci G, J Nonnewton Fluid Mech, 62, 279 (1996).

3) Marrucci G, Ianniruberto G, Macromolecules, 37, 3934 (2004).

4) Wagner $\mathrm{MH}$, Kheirandish S, Hassager O, J Rheol, 49, 1317 (2005).

5) Bhattacharjee PK, Oberhauser JP, McKinley GH, Leal LG, Sridhar T, Macromolecules, 35, 10131 (2002).

6) Bhattacharjee PK, Nguyen DA, McKinley GH, Sridhar T, $J$ Rheol, 47, 269 (2003).

7) Ye X, Larson RG, Pattamaprom C, Sridhar T, J Rheol, 47, 443 (2003).

8) Bach A, Almdal K, Rasmussen HK, Hassager O, Macromolecules, 36, 5174 (2003).

9) Nielsen JK, Rasmussen HK, Hassager O, McKinley GH, $J$ Rheol, 50, 453 (2006).

10) Yaoita T, Isaki T, Masubuchi $Y$, Watanabe H, Ianniruberto G,
Marrucci G, Macromolecules, 45, 2773 (2012).

11) Ianniruberto G, Brasiello A, Marrucci G, Proc 7th Annu Eur Rheol Conf, 61 (2011).

12) Masubuchi Y, Takimoto J, Koyama K, Ianniruberto G, Marrucci G, Greco F, J Chem Phys, 115, 4387 (2001).

13) Yaoita $T$, Isaki $T$, Masubuchi $Y$, Watanabe $H$, Ianniruberto $G$, Marrucci G, Macromolecules, 44, 9675 (2011).

14) Nielsen JK, Rasmussen HK, Hassager O, J Rheol, 52, 885 (2008).

15) Pearson DS, Kiss AD, Fetters LJ, Doi M, J Rheol, 33, 517 (1989).

16) Janeschitz-Kriegl H, Polymer Melt Rheology and FlowBirefringence, Springer Berlin / Heidelb (1983).

17) Takeda K, Sukumaran SK, Sugimoto M, Koyama K, Masubuchi Y, Nihon Reoroji Gakkaishi (J Soc Rheol Jpn), 43, 63 (2015).

18) Masubuchi $Y$, Matsumiya $Y$, Watanabe $H$, Marrucci $G$, Ianniruberto G, Macromolecules, 47, 3511 (2014).

19) Masubuchi $Y$, Matsumiya $Y$, Watanabe $H$, Macromolecules, 47, 6768 (2014).

20) de Gennes PG, J Chem Phys, 55, 572 (1971).

21) Doi M, Edwards SF, J Chem Soc Faraday Trans 2, 74, 1789 (1978).

22) Doi M, J Polym Sci Polym Phys Ed, 21, 667 (1983).

23) de Cloizeaux J, Macromolecules, 23, 4678 (1990).

24) Tsenoglou C, ACS Polym Prepr, 28, 185 (1987).

25) Graessley WW, Adv Polym Sci, 47, 67 (1982).

26) Desai PS, Larson RG, J Rheol, 58, 255 (2014).

27) Marrucci G, Grizzuti N, Gazz Chim Itali, 118, 179 (1988).

28) Ianniruberto G, Macromolecules, 48, 6306 (2015).

29) Luap C, Müller C, Schweizer T, Venerus DC, Rheol Acta, 45, 83 (2005). 